\title{
Research
}

\section{The Effect of a Pathologists Experience on Sample Adequacy in Endoscopic Ultrasonography-Guided Fine Needle Aspiration (EUS- FNA): Another Factor to Consider?}

\author{
Shruti Mony ${ }^{*}$, Andrea Pagan ${ }^{2}$, Sajiv Sethi ${ }^{2}$, Ambuj Kumar ${ }^{2}$, and Pushpak Taunk ${ }^{2}$ \\ ${ }^{1}$ Division of Gastroenterology and Hepatology, Johns Hopkins University, USA \\ ${ }^{2}$ Department of Internal Medicine, University of South Florida College of Medicine, USA
}

${ }^{*}$ Correspondence to: Dr. Shruti Mony; Division of Gastroenterology and Hepatology, Johns Hopkins Hospital Zayed Bldg M2058, 1800 Orleans Street, Baltimore, MD 21287, USA; Tel: 410-502-3699; Fax: 4436838335; E-mail: shruti.mony@gmail.com

Received: Oct 22 $2^{\text {nd }}, 2020 ;$ Accepted: Oct 27 ${ }^{\text {th }}, 2020 ;$ Published: Nov 2 $2^{\text {nd }}, 2020$

Citation: Mony S, Pagan A, Sethi S, Kumar A, and Taunk P. The effect of a pathologists experience on sample adequacy in endoscopic ultrasonography-guided fine needle aspiration (EUS- FNA): Another factor to consider? Gastro Open A Open J. 2020; I(1): 11-16

\begin{abstract}
\section{Background}

Endoscopic Ultrasonography-guided Fine Needle Aspiration (EUS-FNA), which is the preferred method for sampling gastrointestinal malignancies, is dependent on multiple factors. We aimed to evaluate if the experience of the on-site pathologist had an effect on the sample adequacy and tissue yield.
\end{abstract}

\section{Methods}

A single center, retrospective cohort study was conducted among patients undergoing EUS-FNA between 2015 to 2018 for solid GI lesions. Sample adequacy, pathologist experience, needle passes, size and cytological diagnosis was collected. On-site pathologist reported the sample adequacy.

\section{Results}

A total of 163 patients (47.2\% male), median age 68 years, with solid GI lesions (79.8\% pancreatic masses), were included. There was no significant correlation between the experience of pathologists and the number of EUS-FNA passes required to attain sample adequacy $(\mathrm{r}=0.158, p=$ 0.078). The mean number of passes to attain adequacy was not statistically significant between pathologists with $<=10$ years of experience versus those with $>10$ years of experience (1.94 vs. 1.97 passes). However, more experienced pathologists requested a greater number of EUS-FNA passes for an adequate sample compared to less experienced pathologists $(4.05 \mathrm{vs.} 3.23 ; p=0.003)$.

\section{Conclusions}

While there was no difference in determining sample adequacy based on pathologist experience, the experienced pathologists actually requested more FNA passes than less experienced ones. One rationale is that younger graduates have better exposure to EUS-FNA, leading to a greater level of confidence with securing a diagnosis. Further data is required to evaluate this effect.

Keywords: Endoscopic Ultrasound; Pathologist Experience; Fine Needle Aspiration; Sample Adequacy; Diagnostic Yield. 


\section{INTRODUCTION}

Endoscopic ultrasound along with fine-needle aspiration (EUS-FNA) is a rapidly evolving method of tissue acquisition since it was first reported nearly 25 years ago. ${ }^{1-3}$ It has emerged as the test of choice and the primary technique for the acquisition in otherwise difficult to access anatomic locations within the abdomen, specifically the pancreas, subepithelial lesions and other structures adjacent to the GI tract. Additionally it is useful for lesions in the retroperitoneum, mediastinum, and peri-rectal space. ${ }^{4}$ Obtaining an adequate sample and reaching an accurate diagnosis are fundamental endpoints of EUS-FNA. The diagnostic accuracy of EUS-FNA for pancreatic lesions is well established in the literature and is reported as up to $90 \% .{ }^{5,6}$ Thus, it has proven to be a minimally invasive, cost effective and a relatively safe procedure with a complication rate of $0.5 \%$ to $2.9 \%{ }^{7,8}$

Despite it being a popular choice among advanced endoscopists, its accuracy and effectiveness are dependent on several key components. These include operator dependent factors (skill of the endoscopist, sufficient yield and accurate targeting), pathologist dependent factors (interpretation and diagnosis by pathologists), presence or absence of on-site cytopathologists and technical factors (lesion site, size, needle type, number of passes, aspiration and expulsion technique). ${ }^{3,9}$

While some studies on the availability of Rapid On-Site Evaluation (ROSE) during EUS-FNA have highlighted their presence as being beneficial others report otherwise. ${ }^{9-15}$ The advantage of ROSE is that the quality and adequacy of the sample obtained by the endosonographer can be interpreted during the procedure by a cytopathologist who can also provide a preliminary diagnosis. Thus, reducing the sampling error, yield and overall number of FNA passes. It is reported to be associated with a $10 \%$ to $15 \%$ rise of diagnostic yield in the EUS performance for pancreatic and non-pancreatic lesions. ${ }^{15}$ These conflicting results in diagnostic yield are likely due to the aforementioned factors that influence diagnostic yield, including the operator dependent and technical factors for tissue acquisition. Additionally, not all centers have easy accessibility to on site cytopathologists.

In regard to needle sizes, randomized studies and meta-analysis have demonstrated that there is no significant difference between $22 \mathrm{G}$ and $25 \mathrm{G}$ needles used during EUS-FNA for the diagnosis of solid pancreatic lesions. ${ }^{16}$ Although $25 \mathrm{G}$ needles may have an added advantage of adequacy there was no difference in accuracy, number of passes, or complications compared to $22 \mathrm{G}$ needles in the evaluation of pancreatic and peri-pancreatic lesions. ${ }^{16-18}$

We have identified several studies that have addressed other operator dependent and technical factors affecting the diagnostic accuracy for EUS-FNA including suction, slow pull, and fanning technique. However, to date, there have been no published studies that assess the experience of a pathologist on the number of FNA passes and sample adequacy. The aim of our study was to evaluate if the experience of the on -site pathologist had an effect on the sample adequacy and tissue yield, specifically on the number of passes needed for adequacy. We hypothesized that the more experienced pathologist would be more likely to deem a sample adequate and require lesser number of FNA passes for diagnostic evaluation.

\section{MATERIALS \& METHODS}

\section{Study design}

We performed a retrospective cohort study of all consecutive outpatients and inpatients who underwent EUS-FNA of solid GI lesions at a single tertiary referral hospital (Tampa General Hospital, Tampa, Florida) between March $1^{\text {st }} 2011$ to December $31^{\text {st }} 2018$. The study was approved by the local institutional review committee at Tampa General Hospital and the University of South Florida [IRB\#: Pro00038813, Date: 8/27/2019].

\section{Patient Characteristics}

All consecutive patients 18 years and older who underwent EUS-FNA of solid GI lesions and lymph nodes concerning for malignancy between March $1^{\text {st }} 2011$ to December $31^{\text {st }} 2018$ who required an on-site cytopathological evaluation (ROSE) were included in the analysis. EUS procedures were reviewed in the electronic health record.

We excluded patients whose information regarding the procedure was incomplete and those who were lost to follow-up and for whom sufficient information to establish the final diagnosis was unavailable. Additionally, patients who had pancreatic cystic lesions or gastrointestinal stromal tumors were excluded as these lesions typically have limited cytological yield and we do not routinely use ROSE for these lesions. Thus, these lesions and those for which there was no availability of ROSE were excluded from the study.

\section{Procedural Technique}

All EUS-guided tissue acquisition procedures were performed by four experienced operators, each of whom had performed more than a 1000 EUS-guided tissue acquisitions. The procedures were performed using Propofol with monitored anesthesia care.

The procedures were performed using The Olympus TJFQ180V, linear echoendoscope. The needles used included 22G and $25 \mathrm{G}$ cytology and histology needles. The selection of the needle and the number of passes was at the endosonographer's discretion. All procedures were performed by first localizing the target lesion on EUS and confirming the absence of intervening vessels via color flow Doppler. A stylet was routinely used when puncturing. Further needle passes and FNA technique, either slow pull or suction, were performed at the discretion of the endosonographer and on-site pathologist after gross visual assessment of the initial specimen. All procedures were performed in the presence of an on-site pathologist. The on-site samples were fixed in CytoLyt, Thinprep, air dried and also placed in 2 alcohol fixed slides. The on-site pathologist would assess for sample adequacy based on each needle pass. These were reported as adequate or inadequate. In some cases, a preliminary diagnosis would be provided which was recorded in the patient's pathology report.

All adverse events following the procedure were documented.

\section{Data Collection and Final Diagnosis}

The data collected from the endoscopy reports included: demographic variables (age and sex of the patient), target lesion requiring EUS- FNA needle type ( $22 \mathrm{G}$ or $25 \mathrm{G}$ ), number of EUS-FNA passes. The data collected from the pathology reports included: preliminary specimen adequacy status, experience of pathologist performing ROSE and final pathological diagnosis.

The target lesion was categorized based on location: pancre- 
atic lesions (head, uncinate process, tail, body, genu) and non-pancreatic lesions (biliary, lymph nodes, liver).

Cytopathologists performing ROSE were divided into 2 groups, based on their years of experience since graduating from residency as less than 10 years and more than 10 years of experience calculated by the median years of experience.

The final tissue diagnosis was made after the slides were assessed by either a different or same pathologist, for confirmation of the specimen. Only lesions whose final pathological diagnosis was positive for malignancy were considered.

Inconclusive, benign and negative cytology were not included. Cystic lesions and those concerning for gastrointestinal stromal tumors were also excluded due to the need for histologic tissue acquisition and false negative rate. ${ }^{19}$

\section{Outcomes}

The primary outcome was correlation between number of passes required to attain an adequate EUS-FNA samples and the experience of the on-site pathologist. Sample adequacy was defined as ability to procure cytologic/ histologic samples adequate for interpretation. Each FNA pass requested by the cytopathologist was deemed either adequate or inadequate. The adequacy of each sample obtained from a pass was determined by the cytopathologist in real time. The final diagnosis was determined based on a combination of surgical pathology; biopsy of primary tumor or metastatic lesions; serial imaging; labs such as tumor markers; and clinical course.

Secondary outcomes included correlation of sample adequacy and pathologists' experience according to needle type, target location, procedure time and adverse events. We also assessed the association between pathologist experience and sample adequacy by categorizing the experience as $<=10$ years versus more than 10 years.

\section{Statistical Analysis}

The demographic and procedure variables were summarized as mean and standard deviation for continuous variables and rates for categorical variables. ${ }^{20}$ The correlation between pathologist experience and EUS-FNA sample adequacy was summarized using the non-parametric Spearman's rank correlation coefficient. The association between adequacy of EUS-FNA sample according to experience of pathologist (binary) was assessed using binary logistic regression and summarized as odds ratio (OR) along with 95\% Confidence Intervals (CI).

A p-value of $<0.05$ was considered statistically significant. All analyses were performed using IBM SPSS statistical analysis software (BM Corp. Released 2017. IBM SPSS Statistics for Windows, Version 25.0. Armonk, NY: IBM Corp.).

\section{RESULTS}

\section{Clinical Characteristics}

A total of 163 patients with solid GI lesions suspicious for malignancy underwent EUS-FNA between March 2011 to December 2018. The median age was 68 years, ranging from 31 years to 96 years. A total of $47.2 \%(n=77)$ were male. A total of $79.8 \%(n=130)$ target lesions were solid pancreatic lesions and the remainder were non-pancreatic lesions. Among the various needles used, 25G needles were used in 35\% ( $n=57)$ cases and $22 \mathrm{G}$ needles in $65 \%(n=106)$ of cases. (Table 1$)$
Table 1. Patient characteristics undergoing EUS-FNA

\begin{tabular}{|l|l|l|}
\hline Variables & Total Number & \% \\
\hline Overall & $\mathrm{N}=163$ & \\
\hline Age(years), mean years & $67.44(12.6)$ & \\
\hline Males & 77 & 47.2 \\
\hline Females & 86 & 52.8 \\
\hline Needle type & \multicolumn{2}{|l|}{} \\
\hline $25 \mathrm{G}$ & 57 & 35 \\
\hline $22 \mathrm{G}$ & 106 & 65 \\
\hline Target Lesion & \multicolumn{2}{|l|}{} \\
\hline Pancreatic & 130 & 79.8 \\
\hline Non-Pancreatic & 33 & 20.2 \\
\hline
\end{tabular}

\section{Pathologist Experience}

There was a total of 16 pathologists who were involved in on-site evaluation of EUS-FNA specimens. Of these, their mean years of experience was 14 years and median year of experience 10 years. The cut off for less experienced pathologist was taken as less than or equal to 10 years and that for more experienced pathologists was greater than 10 years. The range of experience extended from 3 years of experience to a maximum of 46 years of experience.

Correlation between Pathologist Experience and number of passes for sample adequacy: As shown in, there was a very weak correlation between pathologist years of experience and the pass at which the sample was deemed adequate (correlation coefficient $=0.158$, $p=0.078$ ) as shown in the graph in Figurel. The correlation between pathologist years of experience and the pass at which the sample was deemed adequate according to needle size was also very weak. The correlation coefficient was $0.121(p=0.429)$ for the $25 \mathrm{G}$ needle and 0.131 for the $22 \mathrm{G}$ needle $(p=0.248)$. The correlation was weak for non-pancreatic cancers (correlation coefficient $=0.4 ; p=0.035$ ) and very weak for pancreatic cancers (correlation coefficient $=0.105 ; p=0.308$ ).

Figure 1. Scatter plot illustrating the correlation between number of passes ( $y$ axis) and pathologist experience ( $\mathrm{x}$ axis).

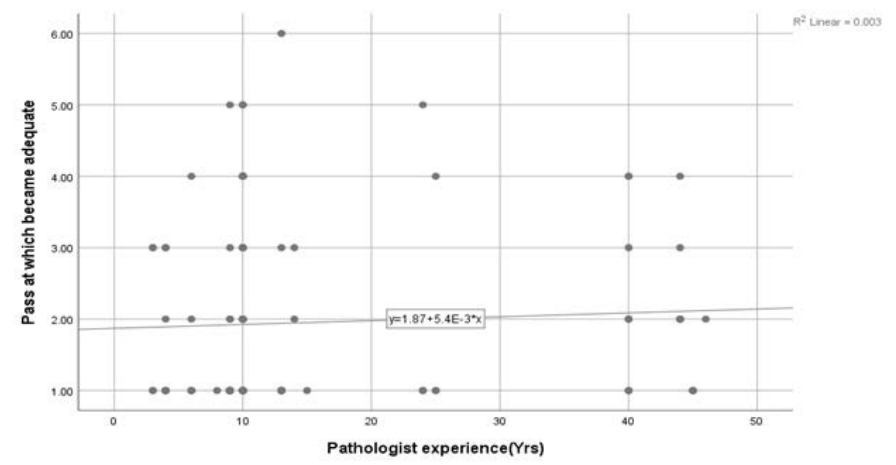

Association between pathologist experience and final adequacy of EUS-FNA sample: The difference in adequacy rate was not statistically significant between pathologists with $<=10$ years of experience $(77 \%)$ versus pathologists with $>10$ years of experience $(76 \%)$. The 
unadjusted OR was 1.01 (95\% CI 0.47 to $2.22 ; p=0.965$ ). The findings remained unchanged when adjusted for needle size and location (pancreatic versus non-pancreatic). The adjusted odds ratio was 0.978 (95\% CI 0.44 to $2.15 ; p=956$ ).

Difference in number of passes required for adequacy and pathologist experience: The mean number of passes to reach adequacy was not significantly different between pathologists with $<=10$ years of experience (1.94 passes \pm 1.25 ) versus $>10$ years of experience $(1.97 \pm 1.3)$ resulting in a mean difference of 0.32 ( $95 \%$ CI -0.52 to 0.46 ; $p=0.826$ ). The results remained unchanged regardless of needle size or location (pancreatic versus non-pancreatic).

Number of passes needed for an adequate versus inadequate sample and pathologist experience: Overall, the mean number of passes for an adequate sample was $3.49( \pm 1.38)$, which was significantly lower, versus $4.13( \pm 1.47)$ for an inadequate sample $(p=0.014)$. While the difference in number of passes needed for an adequate versus inadequate sample was statistically significant for the $25 \mathrm{G}$ needle $(3.13$ $[ \pm 1.35]$ for adequate sample versus $4.17[ \pm 0.718]$ for an inadequate sample; $p=0.008)$, the difference was not statistically significant for the $22 \mathrm{G}$ needle (3.69 [ \pm 1.37$]$ for adequate sample versus $4.12[ \pm 1.7]$ for inadequate sample; $p=0.194)$. Similarly, the difference in number of passes needed for an adequate versus inadequate sample was statistically significant for pancreatic $(3.55[ \pm 1.31]$ for adequate sample versus 4.27 [ \pm 1.38 ] for an inadequate sample; $p=0.009$ ) but not for non-pancreatic (3.29 $[ \pm 1.63]$ for adequate sample versus $3.2[ \pm 1.92]$ for inadequate sample; $p=0.898$ ). The mean number of passes for an adequate sample by pathologists with $<=10$ years of experience was $3.23( \pm 1.3)$, which was significantly lower, versus $4.05( \pm 1.43)$ by pathologists with $>10$ years of experience $(p=0.003)$.

\section{DISCUSSION}

Endoscopists are cognizant of the multiple factors affecting the effectiveness of the EUS-FNA procedure. A factor that has been debated over the years is the presence of ROSE by cytopathologists. Several studies initially reported it as enhancing the sample adequacy, diagnostic yield and requiring fewer needle passes. ${ }^{9,10,12,21,22}$ More recent meta-analysis and randomized controlled trials also indicated that there was a reduction in required needle passes, but overall no benefit was established on diagnostic yield, proportion of adequate specimens and accuracy in pancreatic masses. ${ }^{13-15,23}$ Thus, ROSE is not rendered mandatory for this purpose. At our institution (Tampa General Hospital) we have the availability of experienced on- site pathologist and thus have their presence in all our cases suspicious for malignancy requiring EUS-FNA, which was incorporated in our study. While utilizing the benefit of ROSE, a key component to keep in mind is the expertise of the endosonographer and the on-site cytopathologists. This in turn may affect the final diagnostic yield.

Our retrospective study is the first of its kind in evaluating the effect of a pathologist experience on sample adequacy. Our findings show that there is no significant correlation between the experience of pathologists and the number of passes required to attain adequacy of EUS-FNA sample. Furthermore, the results remained essentially unchanged when adjusted for needle size and location (pancreatic versus non-pancreatic). The difference in adequacy rate was not statistically significant between pathologists with $<=10$ years of experience $(77 \%)$ versus pathologists with $>10$ years of experience (76\%), neither was the mean number of passes required to attain adequacy $<=10$ years of experience (1.94 passes \pm 1.25 ) versus $>10$ years of experience $(1.97 \pm 1.3)$. This suggests that regardless of experience, cytopathologists are equally confident in deeming a sample adequate. This finding was contrary to our initial hypothesis that more experienced pathologists would be more likely to deem a sample adequate given their increased exposure and patient volume. One plausible rationale for our results is that given the significant growth of EUS-FNA in the last 2 decades, less experienced pathologists may actually have more exposure to EUS-FNA samples and better formal training.

However, we noted that the pathologists with greater than 10 years of experience requested a greater number of EUS-FNA passes for the target lesion for an adequate sample compared to the less experienced pathologist $4.05( \pm 1.43)$ vs. $3.23( \pm 1.3)$, which was statistically significant $(p=0.003)$. Thus, while there was no difference in determining adequacy of a sample based on pathologist experience, more experienced pathologists actually requested a greater number of FNA passes than less experienced pathologists. Again, this was contrary to our initial hypothesis in that more experienced pathologists should request less FNA passes given their increased exposure to the process of on-site cytopathology. The significant growth in volume of EUS-FNA has likely provided younger graduates with excellent exposure and led to a greater level of confidence with securing a diagnosis.

Our study was compatible with the data reported in literature, in that the average number of EUS-FNA passes for solid lesions is a mean of 3-4. ${ }^{24-26}$ In regard to FNA needle gauge, our endosonographers primarily used the $22 \mathrm{G}$ and $25 \mathrm{G}$ needles. Overall, there was no correlation with needle gauge, sample adequacy and the years of pathologist experience. This was in line with previous studies. ${ }^{17,27,28}$ In the presence of ROSE, 19G EUS-FNA needles did not confer a diagnostic advantage over the more commonly used 22 and $25 \mathrm{G}$ needles with respect to diagnostic accuracy, number of passes, and complications. ${ }^{29}$

There are multiple advantages to our study. We included a wide spectrum of lesions in addition to pancreatic malignancies. The non-pancreatic lesions being-lymph nodes, metastatic lesions in the liver, adrenal and retroperitoneal masses. We only included lesions in which the final pathological diagnosis was consistent with malignancy or those in which there were significant atypical cells and the overall clinical picture (based on imaging, labs, surgical specimen) was consistent with malignancy, thus eliminating samples which were partly inconclusive or benign lesions. We utilized ROSE pathologist at our center to determine sample adequacy. The final pathological diagnosis was read by a different pathologist than the one present on site, reducing the possibility of sample bias.

The procedure was performed safely and efficiently. There were no adverse events during or immediately following the procedure highlighting EUS-FNA to be a safe intervention with a low post-procedural adverse events rate. ${ }^{7,30}$

The limitations of our study are varied. First, it was retrospective, single centered with a relatively small sample size. Second, there is no standardized definition on the adequacy of a sample and that was left to the discretion of the on-site pathologist. Sampling cells from a lymph node and pancreatic mass may be easier compared to other lesions. Thus, adequacy cannot be defined equivalently across different organs and organ systems and further studies are needed to define the 
precise number of cells required to label a slide positive for malignancy We did not consider the details of sample preparation, which are factors that may have contributed to sample adequacy. Lastly, none of our pathologists had formal training on GI pathology. It is also difficult to interpret the expertise and skill of the pathologist based solely on years since graduating from residency. Further prospective studies are needed to investigate ideal sample preparation to maximize the diagnostic yield of ROSE, and also to determine the optimal number of FNA passes to perform during this procedure.

An obvious limitation of this study is that each sample was evaluated by either a more experienced or less experienced cytopathologist, but never both. Thus, there was no direct head-to-head comparison for a particular sample between pathologists with varying degrees of experience. This type of evaluation would not be possible in a retrospective study and prospectively, would require significant time and cost. Finally, sample adequacy may also be dependent on the endosonographer skill level and the quality of specimens obtained. Given that the majority of FNAs were performed by two endosonographers with the same number of years of training, the effect of endosonographer skill level was unlikely to affect the final results.

We hoped to shed light on the prospect of the experience of a pathologist on the efficacy and adequacy of an EUS-FNA sample. Future studies are warranted that incorporate a more standardized method to assess sample adequacy, cost analysis, procedure time and the experience of an endoscopist. Current methods in sample preparation for ROSE may also need to change to optimize the detection of malignant cells during this procedure. Lastly, one must consider newer modalities such as EUS-guided core biopsy that may soon replace EUS-FNA as it is reported to have comparable pathologic diagnostic yield to EUSguided FNA with fewer passes required and better yield in the absence of ROSE. ${ }^{31}$

\section{CONCLUSION}

Although the future remains promising, current procedural practices are dictated by numerous technical factors including needle availability and cost, lesion location and type, sample adequacy, pathologist and endosonographer's experience and preferences. Our study showed that ROSE is accurate regardless of the experience of a pathologist. Further research is required to validate these findings.

\section{CONFLICTS OF INTEREST}

None.

\section{REFERENCES}

1. Wani S, et al. Diagnostic yield of malignancy during EUS-guided FNA of solid lesions with and without a stylet: A prospective, single blind, randomized, controlled trial. Gastrointest Endosc. 2012; 76(2): 328-335. doi: 10.1016/j.gie.2012.03.1395

2. DiMagno EP, et al. Ultrasonic endoscope. Lancet. 1980; 1(8169): 629631. doi: 10.1016/s0140-6736(80)91122-8.

3. Cazacu IM, et al. A quarter century of EUS-FNA: Progress, milestones, and future directions. Endoscopic ultrasound. 2018; 7(3): 141160. doi: 10.4103/eus.eus_19_18

4. Lachter J. Basic technique in endoscopic ultrasound-guided fine nee- dle aspiration for solid lesions: What needle is the best? Endosc Ultrasound. 2014. 3(1): 46-53. doi: 10.4103/2303-9027.124313

5. Chen G, et al. Diagnostic accuracy of endoscopic ultrasound-guided fine-needle aspiration for pancreatic cancer: a meta-analysis. Pancreatology. 2013; 13(3): 298-304. doi: 10.1016/j.pan.2013.01.013

6. Baek HW, et al. Diagnostic accuracy of endoscopic ultrasound-guided fine needle aspiration cytology of pancreatic lesions. J Pathol Transl Med. 2015; 49(1): 52-60. doi: 10.4132/jptm.2014.10.26

7. Al-Haddad M, et al. The safety of fine-needle aspiration guided by endoscopic ultrasound: A prospective study. Endoscopy. 2008; 40(3): 204-8. doi: 10.1055/s-2007-995336

8. Bluen BE, et al. Accuracy and quality assessment of eus-fna: A singlecenter large cohort of biopsies. Diagn Ther Endosc. 2012; 2012: 139563. doi: 10.1155/2012/139563

9. Wyse J, et al. Onsite evaluation of endoscopic ultrasound fine needle aspiration: The endosonographer, the cytotechnologist and the cytopathologist. Rev Esp Enferm Dig. 2017; 109(4): 279-283.

10. Schmidt RL, et al. The influence of rapid onsite evaluation on the adequacy rate of fine-needle aspiration cytology: A systematic review and meta-analysis. Am J Clin Pathol. 2013; 139(3): 300-308. doi: 10.1309/ AJCPEGZMJKC42VUP

11. Iglesias-Garcia J, et al. Influence of on-site cytopathology evaluation on the diagnostic accuracy of endoscopic ultrasound-guided fine needle aspiration (EUS-FNA) of solid pancreatic masses. Am J Gastroenterol. 2011; 106(9): 1705-1710. doi: 10.1038/ajg.2011.119

12. Klapman JB, et al. Clinical impact of on-site cytopathology interpretation on endoscopic ultrasound-guided fine needle aspiration. Am J Gastroenterol. 2003; 98(6): 1289-1294. doi: 10.1111/j.15720241.2003.07472.x

13. Alsohaibani F, Girgis S, Sandha GS. Does onsite cytotechnology evaluation improve the accuracy of endoscopic ultrasound-guided fineneedle aspiration biopsy? Can J Gastroenterol. 2009; 23(1): 26-30. doi: $10.1155 / 2009 / 194351$

14. Wani S, et al. The clinical impact of immediate on-site cytopathology evaluation during endoscopic ultrasound-guided fine needle aspiration of pancreatic masses: A prospective multicenter randomized controlled trial. Am J Gastroenterol. 2015; 110(10): 1429-1439. doi: 10.1038/ ajg.2015.262

15. Kappelle WFW, et al. Rapid on-site evaluation during endoscopic ultrasound-guided fine-needle aspiration of lymph nodes does not increase diagnostic yield: A randomized, multicenter trial. Am J Gastroenterol. 2018; 113(5): 677-685. doi: 10.1038/s41395-018-0025-8

16. Guedes HG, et al. A comparison of the efficiency of $22 \mathrm{G}$ versus $25 \mathrm{G}$ needles in EUS-FNA for solid pancreatic mass assessment: A systematic review and meta-analysis. Clinics. 2018; 73: e261-e261. doi: 10.6061/ clinics/2018/e261

17. Affolter KE, et al. Needle size has only a limited effect on outcomes in EUS-guided fine needle aspiration: A systematic review and metaanalysis. Dig Dis Sci. 2013; 58(4): 1026-1034. doi: 10.1007/s10620-0122439-2

18. Vilmann P, et al. Multicenter randomized controlled trial com- 
paring the performance of 22 gauge versus 25 gauge EUS-FNA needles in solid masses. Scand J Gastroenterol. 2013; 48(7): 877-883. doi: 10.3109/00365521.2013.799222

19. Aadam AA, et al. A randomized controlled cross-over trial and cost analysis comparing endoscopic ultrasound fine needle aspiration and fine needle biopsy. Endosc Int Open. 2016; 4(5): E497-E505. doi: 10.1055/s-0042-106958

20. Kroep S, et al. Comparing trends in esophageal adenocarcinoma incidence and lifestyle factors between the United States, Spain, and the Netherlands. Am J Gastroenterol. 2014; 109(3): 336-343; quiz 335, 344. doi: 10.1038/ajg.2013.420

21. Koul A, et al. The efficacy of rapid on-site evaluation during endoscopic ultrasound-guided fine needle aspiration of pancreatic masses. Gastroenterol Rep (Oxf). 2018; 6(1): 45-48. doi: 10.1093/gastro/gox017

22. Hebert-Magee $S$, et al. The presence of a cytopathologist increases the diagnostic accuracy of endoscopic ultrasound-guided fine needle aspiration cytology for pancreatic adenocarcinoma: A meta-analysis. Cytopathology. 2013; 24(3): 159-171. doi: 10.1111/cyt.12071

23. O'Connor K, et al. Gastrointestinal endoscopic ultrasound-guided fine-needle aspiration biopsy specimens: Adequate diagnostic yield and accuracy can be achieved without on-site evaluation. Acta Cytol. 2015; 59(4): 305-310. doi: 10.1159/000439398

24. Moller K, et al. EUS-guided FNA of solid pancreatic masses: High yield of 2 passes with combined histologic-cytologic analysis. Gastrointest Endosc. 2009; 70(1): 60-69. doi: 10.1016/j.gie.2008.10.008
25. LeBlanc JK, et al. Optimal number of EUS-guided fine needle passes needed to obtain a correct diagnosis. Gastrointest Endosc. 2004; 59(4): 475-481. doi: 10.1016/s0016-5107(03)02863-3

26. Erickson RA, Sayage-Rabie L, Beissner RS. Factors predicting the number of EUS-guided fine-needle passes for diagnosis of pancreatic malignancies. Gastrointest Endosc. 2000; 51(2): 184-190. doi: 10.1016/ s0016-5107(00)70416-0

27. Xu MM, et al. Comparison of two different size needles in endoscopic ultrasound-guided fine-needle aspiration for diagnosing solid pancreatic lesions: A meta-analysis of prospective controlled trials. Medicine (Baltimore). 2017; 96(5): e5802. doi: 10.1097/MD.0000000000005802

28. Varadarajulu S, et al. The 25-gauge EUS-FNA needle: Good for onsite but poor for off-site evaluation? Results of a randomized trial. Gastrointest Endosc. 2014; 80(6): 1056-1063. doi: 10.1016/j.gie.2014.05.304

29. Muniraj T, Aslanian HR. New Developments in Endoscopic Ultrasound Tissue Acquisition. Gastrointest Endosc Clin N Am. 2017; 27(4): 585-599. doi: 10.1016/j.giec.2017.06.008

30. Thomas T, et al. Efficacy, safety, and predictive factors for a positive yield of EUS-guided Trucut biopsy: A large tertiary referral center experience. Am J Gastroenterol. 2009; 104(3): 584-591. doi: 10.1038/ ajg.2008.97

31. DiMaio CJ, et al. Initial experience with a novel EUS-guided core biopsy needle (SharkCore): Results of a large North American multicenter study. Endosc Int Open. 2016; 4(9): E974-E979. doi: 10.1055/s0042-112581. 